\title{
Odd Periodic Solutions of Fully Second-Order Ordinary Differential Equations with Superlinear Nonlinearities
}

\author{
Yongxiang Li and Lanjun Guo \\ Department of Mathematics, Northwest Normal University, Lanzhou 730070, China \\ Correspondence should be addressed to Yongxiang Li; liyx@nwnu.edu.cn
}

Received 17 January 2017; Revised 2 June 2017; Accepted 26 July 2017; Published 23 August 2017

Academic Editor: Lishan Liu

Copyright (c) 2017 Yongxiang Li and Lanjun Guo. This is an open access article distributed under the Creative Commons Attribution License, which permits unrestricted use, distribution, and reproduction in any medium, provided the original work is properly cited.

\begin{abstract}
This paper is concerned with the existence of periodic solutions for the fully second-order ordinary differential equation $u^{\prime \prime}(t)=$ $f\left(t, u(t), u^{\prime}(t)\right), t \in \mathbb{R}$, where the nonlinearity $f: \mathbb{R}^{3} \rightarrow \mathbb{R}$ is continuous and $f(t, x, y)$ is $2 \pi$-periodic in $t$. Under certain inequality conditions that $f(t, x, y)$ may be superlinear growth on $(x, y)$, an existence result of odd $2 \pi$-periodic solutions is obtained via Leray-Schauder fixed point theorem.
\end{abstract}

\section{Introduction and Main Result}

In this paper, we discuss the existence of odd $2 \pi$-periodic solutions for the fully second-order ordinary differential equation

$$
u^{\prime \prime}(t)=f\left(t, u(t), u^{\prime}(t)\right), \quad t \in \mathbb{R}
$$

where the nonlinearity $f: \mathbb{R}^{3} \rightarrow \mathbb{R}$ is continuous and $f(t, x, y)$ is $2 \pi$-periodic with respect to $t$.

The existence of periodic solutions for nonlinear secondorder ordinary differential equations is an important topic in ordinary differential equation qualitative analysis. It has attracted many authors' attention and concern, and the most works are on the special equation

$$
u^{\prime \prime}(t)=f(t, u(t)), \quad t \in \mathbb{R}
$$

which does not contain explicitly first-order derivative term in nonlinearity. Many theorems and methods of nonlinear functional analysis have been applied to the periodic problems of (2), such as the upper and lower solutions method and monotone iterative technique [1-4], the continuation method of topological degree [5-9], variational method and critical point theory [10-14], method of phase-plane analysis [15-19], the Krasnoselskii's type fixed point theorem in cone [20-23], and the theory of fixed point index [24-26].
However, there are not so many existence results for the second-order periodic problem that nonlinearity is dependent on the derivative. See [27-30]. In [27, 28], the authors discussed some special cases that nonlinearity is with a separated derivative term $g\left(u^{\prime}\right)$. By finding the fixed point of the Poincaré mapping, they obtained several existence results. In [29], Hakl et al. considered the second-order periodic problem with linear derivative term

$$
\begin{aligned}
u^{\prime \prime}(t)+f(u(t)) u^{\prime}(t)+g(u(t)) & =h(t, u(t)), \\
t \in[0, \omega], & \\
u(0) & =u(\omega), \\
u^{\prime}(0) & =u^{\prime}(\omega)
\end{aligned}
$$

by the method of lower and upper solutions. In [30], Li and Jiang researched the existence of positive $2 \pi$-periodic solution of the general second-order differential equation (1) by employing fixed point index theory in cones under the nonlinearity $f(t, x, y)$ satisfying a sign condition. Contrary to the results in [30], whether BVP (1) has a sign-changing periodic solution, especially an odd periodic solution, is an interesting problem. For the special second-order ordinary differential equation (2), the existence of odd periodic solutions has been discussed by the present author in [31]. But no one has discussed the general second-order equation (1). The 
main purpose of this paper is to obtain the existence of odd $2 \pi$-periodic solutions for the general second-order equation (1). Our main result is as follows.

Theorem 1. Assume that $f: \mathbb{R}^{3} \rightarrow \mathbb{R}$ is continuous; $f(t, x, y)$ is $2 \pi$-periodic with respect to $t$ and satisfies the following conditions:

(F1) $f(-t,-x, y)=-f(t, x, y), \forall(t, x, y) \in \mathbb{R}^{3}$.

(F2) there exist nonnegative constants $a$ and $b$ satisfying $a+$ $b<1$ and a positive constant $C_{0}$, such that

$$
f(t, x, y) x \geq-a x^{2}-b y^{2}-C_{0}, \quad(t, x, y) \in \mathbb{R}^{3} .
$$

(F3) For any given $M>0$, there is a positive continuous function $g_{M}(\rho)$ on $\mathbb{R}^{+}$satisfying

$$
\int_{0}^{+\infty} \frac{\rho d \rho}{g_{M}(\rho)+1}=+\infty,
$$

such that

$$
\begin{aligned}
& |f(t, x, y)| \leq g_{M}(|y|), \\
& \qquad(t, x, y) \in[0,2 \pi] \times[-M, M] \times \mathbb{R} .
\end{aligned}
$$

Then (1) has at least one odd $2 \pi$-periodic solution.

In Theorem 1, condition (F1) means that $f(t, x, y)$ is an odd function on $(t, x)$. Condition (F2) allows that $f(t, x, y)$ may be superlinear growth on $x$ and $y$. Condition (F3) is a Nagumo type growth condition on $y$, which requests the growth of $f$ on $y$ which cannot be hyperquadric.

The proof of Theorem 1 is based on the Leray-Schauder fixed point theorem, which will be given in the next section. Two examples to illustrate the applicability of our main result are presented at the end of Section 2. Our result and method are different from those in the references mentioned above.

\section{Proof of the Main Result}

Let $C_{2 \pi}(\mathbb{R})$ denote the Banach space of all continuous $2 \pi$ periodic function $u(t)$ in $\mathbb{R}$ with norm $\|u\|=\max _{0 \leq t \leq 2 \pi}|u(t)|$ and $C_{2 \pi}^{n}(\mathbb{R})$ the Banach space of all $n$ th-order continuously differentiable $2 \pi$-periodic functions $u(t)$ in $\mathbb{R}$ with the norm $\|u\|_{C^{n}}=\max \left\{\|u\|_{C},\left\|u^{\prime}\right\|_{C}, \ldots,\left\|u^{(n)}\right\|_{C}\right\}$, where $n$ is a positive integer. Let $L_{2 \pi}^{2}(\mathbb{R})$ be the Hilbert space of locally square integrable $2 \pi$-periodic functions in $\mathbb{R}$ with the interior product $(u, v)=\int_{0}^{2 \pi} u(t) v(t) d t$ and the norm $\|u\|_{2}=$ $\left(\int_{0}^{2 \pi}|u(t)|^{2} d t\right)^{1 / 2}$. Let $H_{2 \pi}^{n}(\mathbb{R})$ be the Sobolev space of $2 \pi$ periodic functions with the norm $\|u\|_{n, 2}=\left(\sum_{i=0}^{n}\left\|u^{(i)}\right\|_{2}^{2}\right)^{1 / 2}$. $u \in H_{2 \pi}^{n}(\mathbb{R})$ means that $u \in C_{2 \pi}^{n-1}(\mathbb{R})$ and $u^{(n-1)}(t)$ is absolutely continuous on any finite interval of $\mathbb{R}$ and $u^{(n)} \in$ $L_{2 \pi}^{2}(\mathbb{R})$. Let $V$ be the subspace of odd functions in $L_{2 \pi}^{2}(\mathbb{R})$. Denote $V^{n}=H_{2 \pi}^{n}(\mathbb{R}) \cap V, W=C_{2 \pi}(\mathbb{R}) \cap V$, and $W^{n}=$ $C_{2 \pi}^{n}(\mathbb{R}) \cap V$. Clearly, $V^{n}$ is a closed subspace of $H_{2 \pi}^{n}(\mathbb{R})$ and hence it is a Banach space by the norm $\|u\|_{n, 2}$ of $H_{2 \pi}^{n}(\mathbb{R}), W^{n}$ is a closed subspace of $C_{2 \pi}^{n}(\mathbb{R})$, and hence it is a Banach space by the norm $\|u\|_{C^{n}}$ of $C_{2 \pi}^{n}(\mathbb{R})$.

Given $h \in V$, we consider the existence of odd $2 \pi$ periodic solution for the linear second-order differential equation

$$
u^{\prime \prime}(t)=h(t), \quad t \in \mathbb{R}
$$

Lemma 2. For every $h \in V$, the linear equation (7) has a unique odd $2 \pi$-periodic solution $u:=S h \in V^{2}$, and it satisfies

$$
\|u\|_{2} \leq\left\|u^{\prime}\right\|_{2}
$$

Moreover, when $h \in W$ the solution $u=S h \in W^{2}$ and $S$ : $W \rightarrow W^{1}$ is a completely continuous linear operator.

Proof. Let $h \in V$. Since $h \in L_{2 \pi}^{2}(\mathbb{R})$ is an odd function, it can be expressed by the Fourier sine series expansion

$$
h(t)=\sum_{k=1}^{\infty} b_{k} \sin k t
$$

where $b_{k}=(2 / \pi) \int_{0}^{\pi} h(s) \sin k s d s, k=1,2, \ldots$, and the Parseval equality

$$
\|h\|_{2}^{2}=\frac{\pi}{2} \sum_{k=1}^{\infty}\left|b_{k}\right|^{2}
$$

holds. It is easy to verify that

$$
u(t)=-\sum_{k=1}^{\infty} \frac{b_{k}}{k^{2}} \sin k t:=\operatorname{Sh}(t)
$$

belongs to $V^{2}$ and is a unique odd $2 \pi$-periodic solution of the linear equation (7) in Carathéodory sense. If $h \in W$, the solution $u \in W^{2}$ is a classical solution. From (11) we can easily see that $S: V \rightarrow V^{2}$ is a linearly bounded operator. By the compactness of the Sobolev embedding $H_{2 \pi}^{2}(\mathbb{R}) \hookrightarrow C_{2 \pi}^{1}(\mathbb{R})$, we see that the embedding $V^{2} \hookrightarrow W^{1}$ is compact. Hence, by the boundedness of the embedding $W \hookrightarrow V, S$ maps $W$ into $W^{1}$ and $S: W \rightarrow W^{1}$ is completely continuous.

On the other hand, since $u^{\prime} \in L_{2 \pi}^{2}(\mathbb{R})$ is an even function, it can be expressed by the cosine series expansion

$$
u^{\prime}(t)=\frac{a_{0}}{2}+\sum_{k=1}^{\infty} a_{k} \cos k t,
$$

where

$$
\begin{aligned}
a_{0} & =\frac{2}{\pi} \int_{0}^{\pi} u^{\prime}(s) d s=u(\pi)-u(0)=0, \\
a_{k} & =\frac{2}{\pi} \int_{0}^{\pi} u^{\prime}(s) \cos k s d s=-\frac{2}{\pi k} \int_{0}^{\pi} u^{\prime \prime}(s) \sin k s d s \\
& =-\frac{2}{\pi k} \int_{0}^{\pi} h(s) \sin k s d s=-\frac{b_{k}}{k}, \quad k=1,2, \ldots
\end{aligned}
$$


Hence we obtain the cosine series expansion of $u^{\prime}$ :

$$
u^{\prime}(t)=-\sum_{k=1}^{\infty} \frac{b_{k}}{k} \cos k t
$$

By the expansions (11) and (14), using Parseval equality, we obtain that

$$
\|u\|_{2}{ }^{2}=\frac{\pi}{2} \sum_{k=1}^{\infty}\left|\frac{b_{k}}{k^{2}}\right|^{2} \leq \frac{\pi}{2} \sum_{k=1}^{\infty}\left|\frac{b_{k}}{k}\right|^{2}=\left\|u^{\prime}\right\|_{2}{ }^{2} .
$$

Hence (8) holds.

Proof of Theorem 1. For every $u \in W^{1}$, set

$$
F(u)(t):=f\left(t, u(t), u^{\prime}(t)\right) .
$$

By the continuity of $f$ and Assumption (F1), $F: W^{1} \rightarrow W$ is continuous and it maps every bounded set of $W^{1}$ into a bounded set of $W$. Define a mapping by

$$
A=S \circ F \text {. }
$$

By Lemma 2, $S: W \rightarrow W^{1}$ is a completely continuous linear operator. Hence the composite mapping $A: W^{1} \rightarrow W^{1}$ is completely continuous. By the definition of the operator $S$, the odd $2 \pi$-periodic solution of (1) is equivalent to the fixed point of $A$. We will use the Leray-Schauder fixed point theorem [32] to show that $A$ has a fixed point. To do this, we consider the homotopic family of the operator equations

$$
u=\lambda A u, \quad 0<\lambda<1 \text {. }
$$

We need to prove that the set of the solutions of the equations (18) is bounded in the space $W^{1}$.

Let $u \in W^{1}$ be a solution of an equation of (18) for $\lambda \in(0,1)$. Then $u=S(\lambda F(u))$. Set $h=\lambda F(u)$. Since $h \in W$, by the definition of $S, u=S h$ is the unique odd $2 \pi$ periodic solution of the linear equation (7). Hence $u \in W^{2}$ satisfies the differential equation

$$
u^{\prime \prime}(t)=\lambda f\left(t, u(t), u^{\prime}(t)\right), \quad t \in \mathbb{R} .
$$

Multiplying this equation by $u(t)$ and using Assumption (F2), we have

$$
\begin{aligned}
u^{\prime \prime}(t) u(t) & =\lambda f\left(t, u(t), u^{\prime}(t)\right) u(t) \\
& \geq \lambda\left(-a(u(t))^{2}-b\left(u^{\prime}(t)\right)^{2}-C_{0}\right) \\
& =-a(u(t))^{2}-b\left(u^{\prime}(t)\right)^{2}-C_{0}, \quad t \in \mathbb{R} .
\end{aligned}
$$

Integrating this inequality on $[0,2 \pi]$, using integration by parts for the left side and (8), we have

$$
\begin{aligned}
-\left\|u^{\prime}\right\|_{2}^{2} & \geq-a\|u\|_{2}^{2}-b\left\|u^{\prime}\right\|_{2}^{2}-2 \pi C_{0} \\
& \geq-(a+b)\left\|u^{\prime}\right\|_{2}^{2}-2 \pi C_{0} .
\end{aligned}
$$

From this inequality it follows that

$$
\left\|u^{\prime}\right\|_{2}^{2} \leq \frac{2 \pi C_{0}}{1-(a+b)} .
$$

By this and (8) we obtain that

$$
\begin{aligned}
\|u\|_{1,2} & \leq\left(\|u\|_{2}^{2}+\left\|u^{\prime}\right\|_{2}^{2}\right)^{1 / 2} \leq \sqrt{2}\left\|u^{\prime}\right\|_{2} \\
& \leq 2\left(\frac{\pi C_{0}}{1-(a+b)}\right)^{1 / 2}:=C_{1} .
\end{aligned}
$$

Hence, by the continuity of the Sobolev embedding $H_{2 \pi}^{1}(\mathbb{R}) \hookrightarrow C_{2 \pi}(\mathbb{R})$, we have

$$
\|u\|_{C} \leq C\|u\|_{1,2} \leq C C_{1}=: M,
$$

where $C$ is the Sobolev embedding constant.

For this $M>0$, by Assumption (F3), there is a positive continuous function $g_{M}(\rho)$ on $\mathbb{R}_{+}$satisfying (5) such that (6) holds. Hence by (6) and (24),

$$
\left|f\left(t, u(t), u^{\prime}(t)\right)\right| \leq g_{M}\left(\left|u^{\prime}(t)\right|\right), \quad t \in \mathbb{R} .
$$

By (5), there exists $M_{1}>M$ such that

$$
\int_{0}^{M_{1}} \frac{\rho d \rho}{g_{M}(\rho)}>2 M .
$$

We use (25) and (26) to show that

$$
\left\|u^{\prime}\right\|_{C} \leq M_{1}
$$

Let $u^{\prime}(t) \neq \equiv$. By the periodicity of $u$, there exist $t_{0} \in$ $(0,2 \pi)$ and $t_{1} \in[0,2 \pi], t_{1} \neq t_{0}$, such that

$$
\begin{aligned}
& u^{\prime}\left(t_{0}\right)=0, \\
& \left\|u^{\prime}\right\|_{C}=\left|u^{\prime}\left(t_{1}\right)\right|>0 .
\end{aligned}
$$

Clearly, one of the following cases holds.

Case 1. $u^{\prime}\left(t_{1}\right)>0, t_{0}<t_{1}$.

Case 2. $u^{\prime}\left(t_{1}\right)>0, t_{0}>t_{1}$.

Case 3. $u^{\prime}\left(t_{1}\right)<0, t_{0}<t_{1}$.

Case 4. $u^{\prime}\left(t_{1}\right)<0, t_{0}>t_{1}$.

We only consider Case 1; the other cases can be dealt with by a similar method. Let Case 1 hold. Set

$$
s_{1}=\sup \left\{s \in\left[t_{0}, t_{1}\right): u^{\prime}(s)=0\right\} .
$$

Then $s_{1}<t_{1}$, and by the definition of supremum,

$$
\begin{aligned}
u^{\prime}(t) & >0, \\
t & \in\left(s_{1}, t_{1}\right] ; \\
u^{\prime}\left(s_{1}\right) & =0 .
\end{aligned}
$$


Hence, for every $t \in\left[s_{1}, t_{1}\right]$, by (19) and (25), we have

$$
\begin{aligned}
u^{\prime \prime}(t) & =\lambda f\left(t, u(t), u^{\prime}(t)\right) \leq \lambda\left|f\left(t, u(t), u^{\prime}(t)\right)\right| \\
& \leq\left|f\left(t, u(t), u^{\prime}(t)\right)\right| \leq g_{M}\left(u^{\prime}(t)\right),
\end{aligned}
$$

so we obtain that

$$
\frac{u^{\prime \prime}(t) u^{\prime}(t)}{g_{M}\left(u^{\prime}(t)\right)} \leq u^{\prime}(t), \quad t \in\left[s_{1}, t_{1}\right] .
$$

Integrating both sides of this inequality on $\left[s_{1}, t_{1}\right]$ and making the variable transformation $\rho=u^{\prime}(t)$ for the left side, we have

$$
\int_{0}^{u^{\prime}\left(t_{1}\right)} \frac{\rho d \rho}{g_{M}(\rho)} \leq u\left(t_{1}\right)-u\left(s_{1}\right) \leq 2\|u\|_{C} \leq 2 M .
$$

From this inequality and (26) it follows that $u^{\prime}\left(t_{1}\right) \leq M_{1}$. Hence, $\left\|u^{\prime}\right\|_{C}=u^{\prime}\left(t_{1}\right) \leq M_{1}$; namely, (27) holds.

Now by (24) and (27), we have

$$
\|u\|_{C^{1}} \leq M_{1} .
$$

This means that the set of the solutions of the equations (18) is bounded in $W^{1}$. By the Leray-Schauder fixed point theorem, $A$ has a fixed point in $W^{1}$, which is an odd $2 \pi$-periodic solution of (1).

The proof of Theorem 1 is completed.

In the above proof, applying the Leray-Schauder fixed point theorem and the technique of prior estimation we proved Theorem 1 . Since the nonlinearity $f(t, x, y)$ may be superlinear growth on $x$ and $y$, Theorem 1 cannot be proved by the simple Schauder fixed point theorem.

Example 3. Consider the superlinear second-order ordinary differential equation

$$
u^{\prime \prime}(t)=u^{3}(t)+u(t)\left(u^{\prime}(t)\right)^{2}+\sin t, \quad t \in \mathbb{R} .
$$

We verify that the corresponding nonlinearity

$$
f(t, x, y)=x^{3}+x y^{2}+\sin t
$$

satisfies the conditions of Theorem 1 . Clearly, $f(t, x, y)$ satisfies conditions (F1) and (F3). For any $(t, x, y) \in \mathbb{R}^{3}$, by definition (36) we have

$$
\begin{aligned}
f(t, x, y) x & =x^{4}+x^{2} y^{2}+x \sin t \geq x \sin t \\
& \geq-\frac{1}{2} x^{2}-\frac{1}{2} \sin ^{2} t \geq-\frac{1}{2} x^{2}-\frac{1}{2}
\end{aligned}
$$

that is, $f$ satisfies condition (F2) for $a=1 / 2, b=0$, and $C_{0}=1 / 2$. Hence $f$ satisfies the conditions of Theorem 1 . By Theorem 1, (35) has at least one odd $2 \pi$-periodic solution.

Example 4. Consider the sublinear second-order ordinary differential equation

$$
u^{\prime \prime}(t)=\sqrt[3]{u(t)} \cos t+\sqrt[3]{u^{\prime}(t)} \sin t+\sin 2 t, \quad t \in \mathbb{R} .
$$

Evidently, the corresponding nonlinearity

$$
f(t, x, y)=\sqrt[3]{x} \cos t+\sqrt[3]{y} \sin t+\sin 2 t
$$

satisfies conditions (F1) and (F3). We show that it also satisfies condition (F2). For any $(t, x, y) \in \mathbb{R}^{3}$, by definition (39),

$$
\begin{aligned}
|f(t, x, y) x| & \leq|x|^{4 / 3}+|y|^{1 / 3}|x|+|x| \\
& \leq \frac{1}{6} x^{2}+\frac{16}{3}+\left(\frac{1}{3}|y|+\frac{2}{3}\right)|x|+|x| \\
& \leq \frac{1}{6} x^{2}+\frac{16}{3}+\frac{1}{6} y^{2}+\frac{1}{6} x^{2}+\frac{5}{3}|x| \\
& \leq \frac{1}{3} x^{2}+\frac{1}{6} y^{2}+\frac{16}{3}+\frac{1}{30} x^{2}+\frac{125}{6} \\
& \leq \frac{11}{30} x^{2}+\frac{1}{6} y^{2}+\frac{157}{6}
\end{aligned}
$$

which is derived by using the Young inequality

$$
A B \leq \frac{1}{p} A^{p}+\frac{1}{q} B^{q}, \quad A, B \geq 0, p, q>1, \frac{1}{p}+\frac{1}{q}=1
$$

to the terms of $|x|^{4 / 3},|y|^{1 / 3},(1 / 3)|y||x|$, and (5/3)|x|. From this inequality it follows that

$$
\begin{aligned}
f(t, x, y) x & \geq-|f(t, x, y) x| \\
& \geq-\frac{11}{30} x^{2}-\frac{1}{6} y^{2}-\frac{157}{6}, \quad(t, x, y) \in \mathbb{R}^{3} .
\end{aligned}
$$

Hence, $f$ satisfies condition (F2). By Theorem 1, (38) has at least one odd $2 \pi$-periodic solution.

Example 3 shows that Theorem 1 is applicable to the superlinear fully second-order ordinary differential equation, and Example 4 shows that Theorem 1 is also applicable to the sublinear fully second-order ordinary differential equation. It should be pointed out that, in Theorem 1 since the nonlinearities contain derivative terms and do not have monotonicity, the conclusions of Examples 3 and 4 cannot be obtained from the known results of [1-31].

\section{Conflicts of Interest}

The authors declare that they have no conflicts of interest.

\section{Acknowledgments}

This research is supported by NNSFs of China (11661071, 11261053, and 11361055).

\section{References}

[1] S. Leela, "Monotone method for second order periodic boundary value problems," Nonlinear Analysis, vol. 7, no. 4, pp. 349355, 1983.

[2] J. J. Nieto, "Nonlinear second-order periodic boundary value problems," Journal of Mathematical Analysis and Applications, vol. 130, no. 1, pp. 22-29, 1988. 
[3] A. Cabada and J. J. Nieto, "A generalization of the monotone iterative technique for nonlinear second order periodic boundary value problems," Journal of Mathematical Analysis and Applications, vol. 151, no. 1, pp. 181-189, 1990.

[4] A. Cabada, "The method of lower and upper solutions for second, third, fourth, and higher order boundary value problems," Journal of Mathematical Analysis and Applications, vol. 185, no. 2, pp. 302-320, 1994.

[5] J.-P. Gossez and P. Omari, "Periodic solutions of a second order ordinary differential equation: a necessary and sufficient condition for nonresonance," Journal of Differential Equations, vol. 94, no. 1, pp. 67-82, 1991.

[6] P. Omari, G. Villari, and F. Zanolin, "Periodic solutions of the Liénard equation with one-sided growth restrictions," Journal of Differential Equations, vol. 67, no. 2, pp. 278-293, 1987.

[7] W. G. Ge, "On the existence of harmonic solutions of Lienard systems," Nonlinear Analysis. Theory, Methods \& Applications, vol. 16, no. 2, pp. 183-190, 1991.

[8] A. Fonda and R. Toader, "Periodic orbits of radially symmetric Keplerian-like systems: a topological degree approach," Journal of Differential Equations, vol. 244, no. 12, pp. 3235-3264, 2008.

[9] D. Tian, "Multiple positive periodic solutions for second-order differential equations with a singularity," Acta Applicandae Mathematicae, vol. 144, pp. 1-10, 2016.

[10] J. Mawhin and M. Willem, "Multiple solutions of the periodic boundary value problem for some forced pendulum-type equations," Journal of Differential Equations, vol. 52, no. 2, pp. 264287, 1984.

[11] V. Coti Zelati, "Periodic solutions of dynamical systems with bounded potential," Journal of Differential Equations, vol. 67, no. 3, pp. 400-413, 1987.

[12] L. Lassoued, "Periodic solutions of a second order superquadratic system with a change of sign in the potential," Journal of Differential Equations, vol. 93, no. 1, pp. 1-18, 1991.

[13] J. Su and L. Zhao, "Multiple periodic solutions of ordinary differential equations with double resonance," Nonlinear Analysis, vol. 70, no. 4, pp. 1520-1527, 2009.

[14] C. Li, R. P. Agarwal, and D. sca, "Infinitely many periodic solutions for a class of new superquadratic second-order Hamiltonian systems," Applied Mathematics Letters. An International Journal of Rapid Publication, vol. 64, pp. 113-118, 2017.

[15] D. Tongren, "Nonlinear oscillations at resonance," Science in China, vol. 12A, pp. 1-12, 1984.

[16] L. Weigu, "A necessary and sufficient condition on the existence and uniqueness of $2 \pi$-periodic solution of Duffing equation," Chinese Annals of Mathematics, vol. 11, no. 3, pp. 342-345, 1990.

[17] A. Fonda and L. Ghirardelli, "Multiple periodic solutions of scalar second order differential equations," Nonlinear Analysis. Theory, Methods \& Applications. An International Multidisciplinary Journal, vol. 72, no. 11, pp. 4005-4015, 2010.

[18] A. Fonda and A. Sfecci, "Periodic bouncing solutions for nonlinear impact oscillators," Advanced Nonlinear Studies, vol. 13, no. 1, pp. 179-189, 2013.

[19] A. Calamai and A. Sfecci, "Multiplicity of periodic solutions for systems of weakly coupled parametrized second order differential equations," NoDEA. Nonlinear Differential Equations and Applications, vol. 24, no. 1, Art. 4, 17 pages, 2017.

[20] F. Merdivenci Atici and G. S. Guseinov, "On the existence of positive solutions for nonlinear differential equations with periodic boundary conditions," Journal of Computational and Applied Mathematics, vol. 132, no. 2, pp. 341-356, 2001.
[21] Y. X. Li, "Positive periodic solutions of nonlinear second order ordinary differential equations," Acta Mathematica Sinica. Chinese Series, vol. 45, no. 3, pp. 481-488, 2002.

[22] P. J. Torres, "Existence of one-signed periodic solutions of some second-order differential equations via a Krasnoselskii fixed point theorem," Journal of Differential Equations, vol. 190, no. 2, pp. 643-662, 2003.

[23] H. Zhu and S. Li, "Multiplicity of positive periodic solutions to nonlinear boundary value problems with a parameter," Journal of Applied Mathematics and Computing, vol. 51, no. 1-2, pp. 245256, 2016.

[24] Y. Li, "Positive periodic solutions of first and second order ordinary differential equations," Chinese Annals of Mathematics. Series B, vol. 25, no. 3, pp. 413-420, 2004.

[25] F. Li and Z. Liang, "Existence of positive periodic solutions to nonlinear second order differential equations," Applied Mathematics Letters. An International Journal of Rapid Publication, vol. 18, no. 11, pp. 1256-1264, 2005.

[26] J. R. Graef, L. Kong, and H. Wang, "Existence, multiplicity, and dependence on a parameter for a periodic boundary value problem," Journal of Differential Equations, vol. 245, no. 5, pp. 1185-1197, 2008.

[27] Z. Wang, "Periodic solutions of the second order differential equations with asymmetric nonlinearities depending on the derivatives," Discrete and Continuous Dynamical Systems. Series A, vol. 9, no. 3, pp. 751-770, 2003.

[28] M. Zamora, "New asymptotic stability and uniqueness results on periodic solutions of second order differential equations using degree theory," Advanced Nonlinear Studies, vol. 15, no. 2, pp. 433-446, 2015.

[29] R. Hakl, P. J. Torres, and M. Zamora, "Periodic solutions of singular second order differential equations: upper and lower functions," Nonlinear Analysis. Theory, Methods \& Applications. An International Multidisciplinary Journal, vol. 74, no. 18, pp. 7078-7093, 2011.

[30] Y. Li and X. Jiang, "Positive periodic solutions for secondorder ordinary differential equations with derivative terms and singularity in nonlinearities," Journal of Function Spaces and Applications, Article ID 945467, 2012.

[31] Y. X. Li, "Oscillatory periodic solutions of nonlinear second order ordinary differential equations," Acta Mathematica Sinica (English Series), vol. 21, no. 3, pp. 491-496, 2005.

[32] K. Deimling, Nonlinear Functional Analysis, Springer, Berlin , Germany, 1985. 


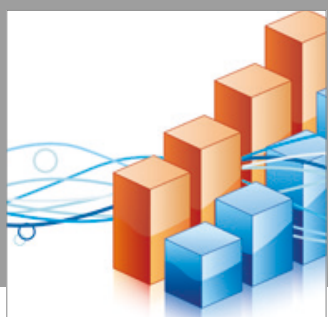

Advances in

Operations Research

vatersals

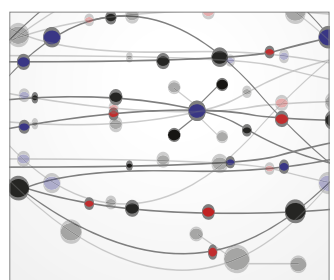

\section{The Scientific} World Journal
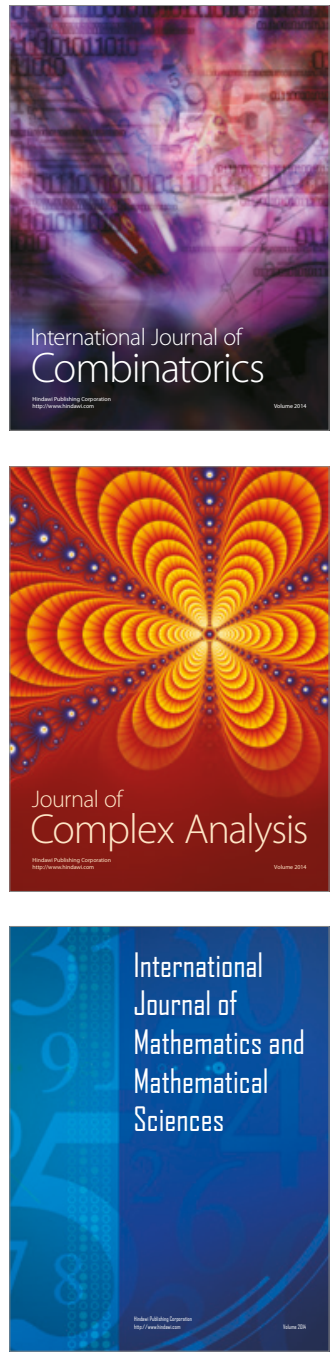
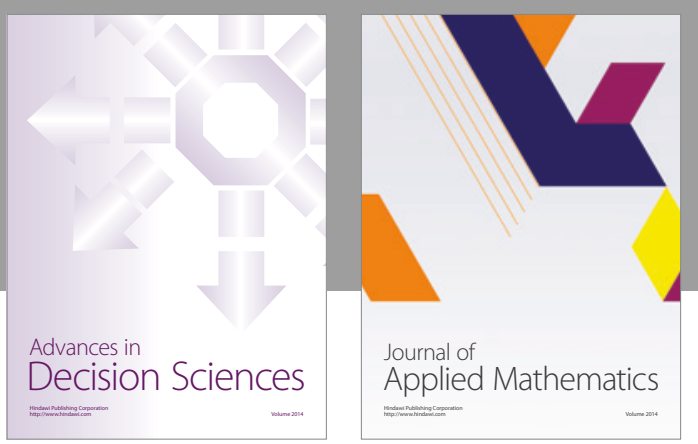

Algebra

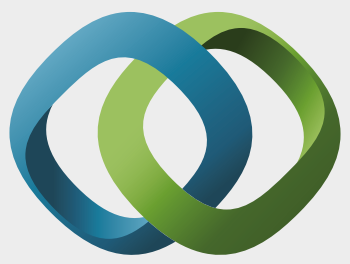

\section{Hindawi}

Submit your manuscripts at

https://www.hindawi.com
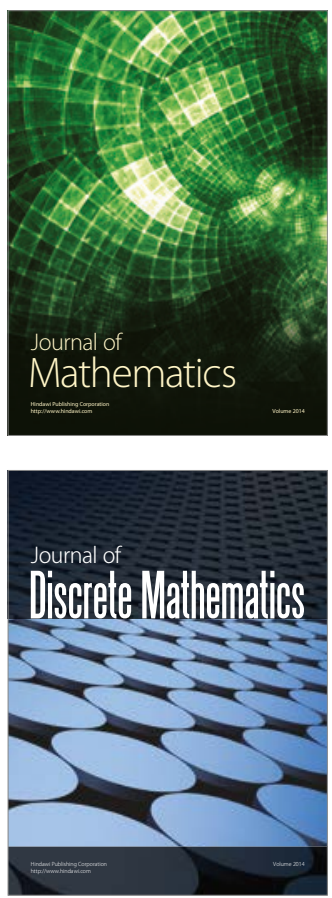

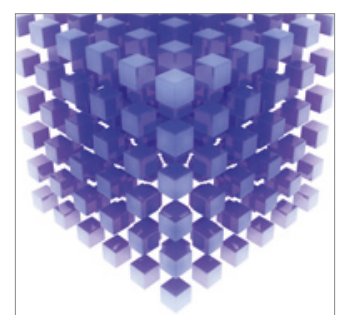

Mathematical Problems in Engineering
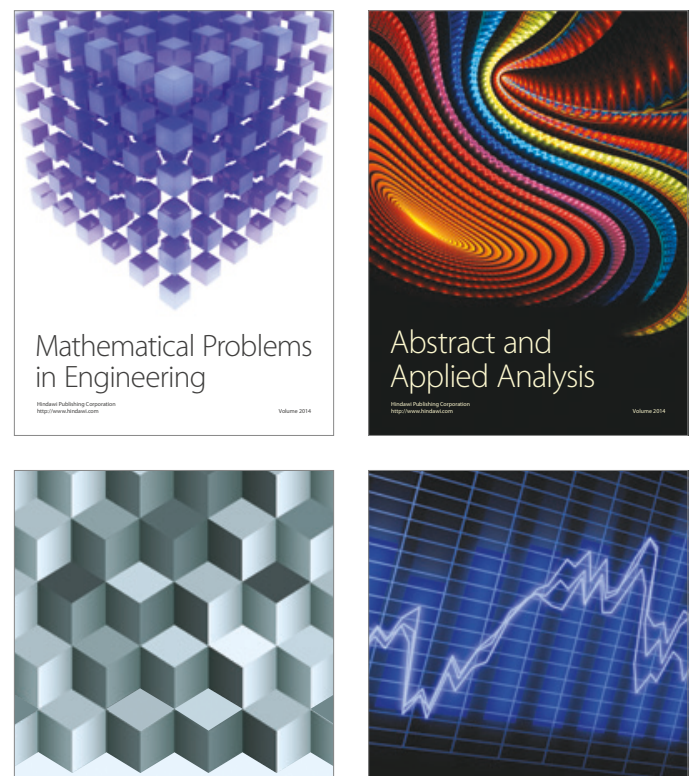

Journal of

Function Spaces

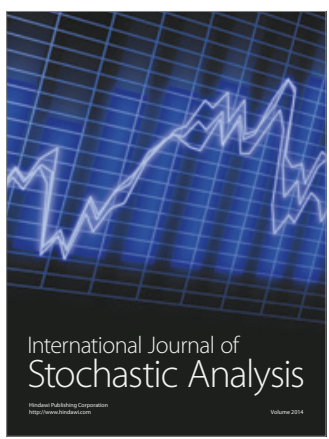

Probability and Statistics
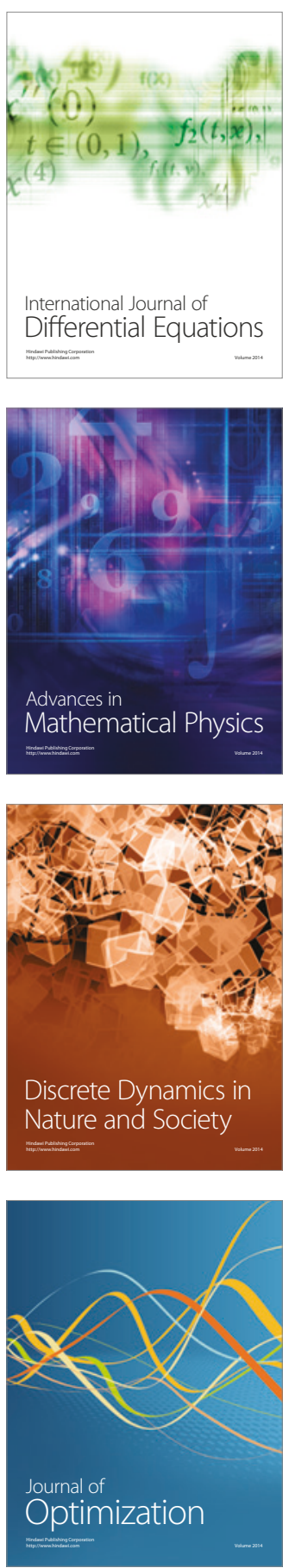